\title{
NIST Transactive Energy Modeling and Simulation Challenge for the Smart Grid-Phase I Report
}

David G. Holmberg

Steven T. Bushby

This publication is available free of charge from:

https://doi.org/10.6028/NIST.TN.2019 


\title{
NIST Transactive Energy Modeling and Simulation Challenge for the Smart Grid-Phase I Report
}

\author{
David G. Holmberg \\ Steven T. Bushby \\ Energy and Environment Division \\ Engineering Laboratory
}

This publication is available free of charge from:

https://doi.org/10.6028/NIST.TN.2019

October 2018

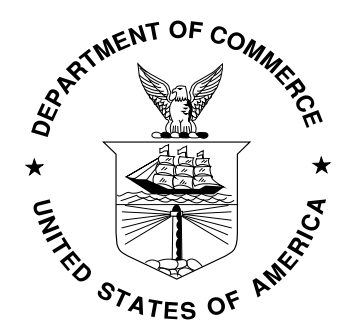

U.S. Department of Commerce Wilbur L. Ross, Jr., Secretary

National Institute of Standards and Technology Walter Copan, NIST Director and Under Secretary of Commerce for Standards and Technology 
Certain commercial entities, equipment, or materials may be identified in this document in order to describe an experimental procedure or concept adequately. Such identification is not intended to imply recommendation or endorsement by the National Institute of Standards and Technology, nor is it intended to imply that the entities, materials, or equipment are necessarily the best available for the purpose.

National Institute of Standards and Technology Technical Note 2019

Natl. Inst. Stand. Technol. Tech. Note 2019, 22 pages (October 2018) CODEN: NTNOEF

This publication is available free of charge from:

https://doi.org/10.6028/NIST.TN.2019 


\begin{abstract}
Phase I of the National Institute of Standards and Technology (NIST) Transactive Energy (TE) Modeling and Simulation Challenge for the Smart Grid spanned September 2015 to September 2016. The TE Challenge was initiated to identify existing simulation tools and expertise that might be developed or combined in co-simulation platforms to enable the evaluation of transactive energy approaches. Seven teams composed of multiple organizations participated in the TE Challenge. This report introduces the teams and summarizes accomplishments from the TE Challenge, which include: definition of TE scenarios and reference grid components; development of a common platform component model for co-simulation; analysis of the TE regulatory environment; analysis of common transactive services; and development of a reference architecture for TE in a microgrid environment.
\end{abstract}

\title{
Key words
}

co-simulation platform; modeling and simulation; smart grid; transactive energy 


\section{Table of Contents}

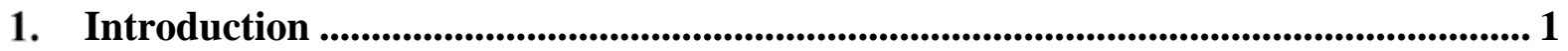

2. TE Challenge Preparations and Launch ........................................................................ 1

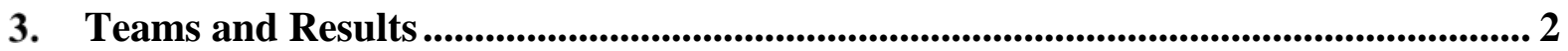

3.1. Business and Regulatory Models Team ……………………………………...... 3

3.2. Reference Grid and Scenarios for TE Simulation Team ........................................... 8

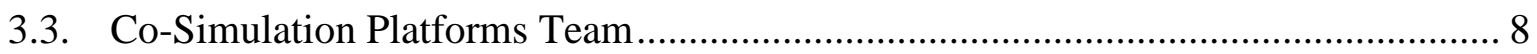

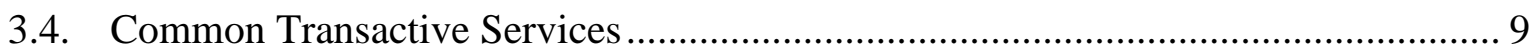

3.5. TE Demo for Microgrid Energy Management Team.................................................. 11

3.6. Transactive Automated Demand Response team ...................................................... 12

3.7. PowerMatcher IoT Team.................................................................................... 13

3.8. Development of a Common TE Abstract Component Model .................................... 13

4. Summary .................................................................................................................................. 16

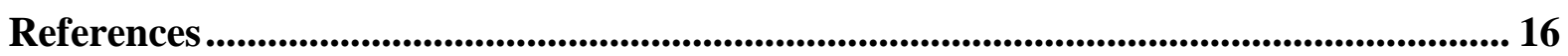




\section{Introduction}

The evolving smart grid, with increased use of renewable energy generation and distributed energy management technologies, offers the potential for significant efficiency improvements through market-based transactive exchanges between energy producers and energy consumers. To understand this potential and support technology developers and policymakers, the smart grid community requires simulation tools and platforms that can be used to explore the benefits and impacts of alternative ways to create and operate transactive energy systems.

The U.S. Department of Energy GridWise Architecture Council (GWAC) has published a Transactive Energy Framework [1] that defines transactive energy broadly as, "a system of economic and control mechanisms that allows the dynamic balance of supply and demand across the entire electrical infrastructure using value as a key operational parameter.” The NIST Transactive Energy Modeling and Simulation Challenge for the Smart Grid (TE Challenge) has brought researchers and companies with simulation tools together with other grid stakeholders to demonstrate modeling and simulation platforms while applying TE approaches to real grid problems.

The goals for Phase I of the TE Challenge were the following:

- Tools - develop/enhance modeling and simulation tools and platforms for TE evaluation;

- Understanding - demonstrate how different TE approaches can improve reliability and efficiency of the electric grid to address today's grid challenges;

- Scenarios - develop a set of scenarios that can serve as ongoing reference points for modeling and simulation;

- Community - create a TE community of people working together and sharing data;

- Progress - produce information that might lead to successful TE pilot projects; and

- Communicate - provide a stage for teams to present their accomplishments and to help industry better understand the capability, repeatability, and utility of simulation platforms that can be used to study the impact of TE approaches.

\section{TE Challenge Preparations and Launch}

NIST hosted a TE Challenge Preparatory Workshop in March 2015. The goal was to solicit input from industry experts on the state-of-the-art for simulation tools and co-simulation platforms that would be valuable for investigating the potential benefits and problems with TE approaches, and to test the proposition that a well-formed industry challenge might stimulate significant advancements. The workshop confirmed the potential value of a TE challenge. This section summarizes the key findings from the preparatory workshop and a subsequent kickoff meeting in September 2015. More details are presented in [2].

The preparatory workshop discussions identified potential simulation tools and provided input to help frame the parameters for the TE Challenge's mission, approach and timeline. The kickoff meeting expanded the number of industry experts involved and helped to refine the TE Challenge parameters. 
In response to questions about simulation tools and platforms, and gaps in the capabilities of these tools, participants identified several existing simulation tools and co-simulation platforms. The list included fourteen commercial and twelve non-commercial tools that model power grids (transmission and distribution level), economics, wholesale power markets, information networks, and buildings. In addition, two platforms to enable cosimulation were noted, along with some standards related to co-simulation. This collection has been shared on the TE Challenge collaboration site Tool Chest [3].

Participants also described some characteristics of co-simulations needed to successfully simulate TE systems. These included: ability to scale to different size physical systems, from feeder level up to city and regional levels; ability to connect physical grid control to financial markets and evaluate stability and security; and ability to model consumer behavior and intelligent agents. Beyond simulation tools, participants also noted the need to better understand business and regulatory models and public policy direction in order to develop more realistic simulations.

In response to questions on the topics of interoperability and metrics, participants provided valuable input. Co-simulation platforms would benefit from sharing a common data model with common data elements and semantics shared at common interfaces. There should be recognized common platform components (e.g., grid, market, customer), and experiments should share common data formats, protocols and reporting.

Common reporting implies metrics for data that are agreed to by different platform developers. Participants identified the need for metrics to capture: cost and benefits for system stakeholders; impact of weather events, power transients, and communication network failure; robustness and stability of TE approaches; and some representative scenarios covering the TE landscape.

In response to questions about how to strengthen the TE Challenge, participants pointed to the need to build a community for collaboration, as well as the need to communicate results to utilities, regulators and lawmakers.

The preparatory workshop and kickoff meeting laid the foundation for the Challenge itself and helped to guide the teams that would carry out the work of fulfilling the Challenge requirements.

\section{Teams and Results}

Seven teams were organized to participate in Phase I of the TE Challenge:

1. Business and Regulatory Models;

2. Reference Grid and Scenarios for TE Simulation;

3. Co-Simulation Platforms;

4. Common Transactive Services;

5. TE Demo for Microgrid Energy Management;

6. Transactive Automated Demand Response (ADR), and

7. PowerMatcher IoT. 
Each team contributed to reaching the goals of the TE Challenge in a different way. The Business and Regulatory Models team sought to understand and communicate the business and regulatory issues impacting possible TE systems. The Reference Grid and Scenarios team focused on the goal of providing a common grid definition for simulation and a set of scenarios that would be useful for comparative simulations (to be later realized in TE Challenge Phase II). The Co-Simulation Platforms team described the key simulation components and interfaces required for any TE co-simulation platform. Interoperable communications between transactive agents was the focus of the TE Demo for Microgrid Energy Management team, as they developed descriptions for a set of TE interactions for microgrid management. The Common Transactive Services team also focused on transactive services, describing a minimal set of common TE market services. The Transactive ADR team initiated an effort within the OpenADR Alliance to incorporate the common transactive services into the established OpenADR protocol to help facilitate future TE implementations. The PowerMatcher IoT team worked to advance a virtual PowerMatcher implementation.

Another result from the Challenge was a cross-cutting effort to develop a common abstract component model to aid the development of co-simulation tools for exploring possible TE approaches. The team membership, goals, and work products are reviewed below.

\subsection{Business and Regulatory Models Team}

\section{Team Members and Goals}

- Leader: John Caldwell, Edison Electric Institute

- Participating organizations: Bluewave Resources, ICF International, CGI Group, Pacific Northwest National Laboratory (PNNL), TeMix Inc., OATI Inc.

- Goals:

o Define fundamental business and regulatory model types;

o Define and characterize the physical and financial interfaces needed to enable participation in TE systems; and

o Identify legislative and regulatory features applicable to each TE approach.

\section{Team Results}

The Business and Regulatory Models team documented the results of their work through two publications: Transactive Energy Models [4] and The Policymaker's Toolkit--Vital Questions to be Addressed about Proposed Transactive Energy Systems [5]. Presentations summarizing these papers were made at the Gridwise Architecture Council TE Systems Conference, Portland, OR, in 2016 and 2017, respectively. These papers are summarized below.

\section{Transactive Energy Models Paper}

In this paper, the team examines the evolving vision of a grid where the utility serves as provider of electricity delivery services and a facilitator of transactions and operations on the grid. The paper begins with a general overview and appraisal of underlying drivers for this transactive grid, and then describes two alternative approaches for transactive systems that are emerging as candidates for putting the vision into operational practice. The paper discusses the evolution of business models that will both enable and be enabled by these transactive systems. It also reviews approaches that are planned or being used to integrate distributed energy resources (DER) around the world. 
The continuing and accelerating growth of DER, specifically photovoltaics (PV), installed each year is identified as a chief driver for change to the electric grid. The growth in DER is attributed to concerns over grid reliability in the context of more extreme weather and aging infrastructure, state and federal level policies that promote development of clean energy, and declining costs for PV systems.

The two main policy tools that support increased use of PV are state renewable portfolio standards and net metering. The paper states that as of 2016, 29 states had passed legislation calling for specific target percentages of non-carbon-emitting energy production in the state. Hawaii set the highest goal of $100 \%$ by 2045 . Typical state targets range from $10 \%$ to $30 \%$ within timeframes of five to ten years. The paper notes that net metering began as a simple means to credit customers for PV energy fed back to the grid, but has become a contentious policy because utilities then pay retail price for customer PV power. Nonetheless, forty-one states and the District of Columbia have net metering policies.

The expansion of DER systems on the distribution grid is also the main driver for considering transactive energy approaches. Notably, the states with the highest levels of DER penetration are also the states exploring new regulatory processes and other means to support improved grid operation. The paper points out that TE fits into the more general topic of "grid modernization.” Grid modernization efforts typically include: grid operations improvements gained through new meter deployments, microgrids, and advanced communications and control; customer engagement through dynamic pricing tariffs and other avenues for active customer interaction; and state incentives or mandates that support grid modernization activities.

The paper notes that local conditions will strongly influence the receptiveness of utilities and regulators to TE systems. Important factors include the existing and projected level of DER penetration, clean-energy goals, the price of electricity, vulnerability to extreme weather events, the economic health of the region, and political considerations. Existing regulatory structures such as vertical integration, the existence of wholesale locational marginal pricing, and the amount of retail customer choice are also important.

The paper presents two alternative approaches for TE systems that could serve areas of the country with wholesale power markets. The first involves a retail market with a two-way subscription tariff implemented using an automated bilateral transaction platform, called TeMix, that is designed to enable peer-to-peer transactions. The transacting party (consumer, prosumer, generator) buys/sells energy and buys associated transport rights in a forward market. These transactions could be as simple as reserving a quantity of energy (and accompanying rights to distribution services) that match the amounts purchased in the previous year. However, the actual energy used on a given day may not match exactly the reserved quantity. Differences between actual energy use and the transacted forward commitment are settled at the real-time market price.

The TeMix model works within the existing tariff regime and does not require a distribution system operator to dispatch resources on the distribution grid. The paper presents a roadmap 
for how the TeMix model could be implemented over the next 15 years in the state of California. A utility pilot project is underway in California to investigate this approach.

The second TE approach makes use of location-based distribution marginal prices (DMP). Current distribution prices are based on the sum of a fee for energy used, a fixed fee, and possibly a demand fee. The DMP would also include an overlay price based on the marginal cost of energy distribution from the substation. The overlay price can be based on long-run (infrastructure planning) or short-run (real-time operational) costs. The paper then discusses the idea of wholesale market location marginal price (LMP) being incorporated such that the total price becomes a combination of LMP and DMP, referred to as "LMP+D".

The challenge to using short-run operational costs is that the infrastructure to determine the costs is not in place. The requirements for such infrastructure include an extensive distribution grid sensor network, high-bandwidth communications, and a cost-effective distributed computing platform to process the information and derive local grid state estimations. The paper presents a roadmap for how DMP might develop gradually over time, beginning with prices based solely on long-run costs and later incorporating short-run costs and distribution system operator dispatch capabilities.

The paper provides a side-by-side analysis of the technical similarities and differences of these two TE approaches for a variety of factors. Both approaches are designed to capture long-term and short-term value. The TeMix approach makes more use of energy markets for managing energy, transport and ancillary services, both for forward and real-time operation. The DMP approach works toward an eventual implementation of a distribution system operator model where distribution resources are bid into a market similar to the wholesale markets today, and then dispatched by the distribution system operator. TeMix has no dispatch; it relies on DER self-dispatch based on forward commitments with discrepancies settled at real-time market prices.

In addition to the technical analysis, the path leading to development of new business models to support transactive approaches is discussed. This analysis begins with an overview of existing electricity business models and the value that each provides. The paper then states that new TE business models may evolve from long-standing current business models, while others may be created specifically for transactive markets. It suggests that revenues will no longer be based on cost of service but rather on the ability to create and capture value. The needs, priorities and decision-making characteristics of consumers will determine their willingness and ability to participate in electricity markets as prosumers or in aggregations of consumers.

The paper explored conditions that will enable TE business models and classes of market participants to emerge over time. The paper authors anticipate that this emergence will result from changes in three mutually-supporting areas: technology penetration; government policy and regulation; and economics. 


\section{The Policymaker's Toolkit: Vital Questions to be Addressed about Proposed Transactive Energy Systems}

The premise of this toolkit is that changes underway in the electric grid compel policymakers to rethink how the grid is managed and regulated. The paper presents several questions that regulators, legislators, and other policymakers will have to contend with if they are confronted with plans or proposals to adopt a transactive energy system. The paper provides a resource to help policymakers determine when and if a transactive energy system is appropriate for the electricity system within their jurisdiction, and how such a system can be most effectively, safely, and equitably implemented.

The topics addressed in this paper were grouped into the following five major topic areas:

- Why Transactive Energy?;

- Managing the Transition to Transactive Energy;

- Market Design;

- Ongoing Market Monitoring and Evaluation; and

- Consumer Protection.

This paper begins with an analysis of trends that are driving the grid toward a more transactive design and then identifies several factors to be considered when evaluating TE systems. Three important trends are:

1) Energy technology advancements including digitization and increased use of automation;

2) Increasingly stringent policy mandates for intermittent utility scale and distributed renewable energy development and energy efficiency; and

3) Changing customer expectations, needs and interests.

Together, these are fundamentally altering electricity sector dynamics. The paper recommends that regulators consider the following factors when evaluating TE system proposals:

- Adherence to sound economic principles, providing cost-reflective pricing, motivating efficient energy usage, and leading consumers to efficient investment in local energy technologies for customer value creation;

- Strategies to cost-effectively capture the potential net benefits that distributed, demand-side resources can offer to the grid, customers and society;

- Energy-efficient and resource-efficient distributed control, coordination and optimization;

- Scalability of the system from home devices to utility scale, coordinating across a system of systems to manage complexity;

- Support for new approaches to distribution system management;

- Ability to grow market participation and thus competition; and

- Use of demonstrations for TE system evaluation.

The paper then presents a series of detailed questions that regulators, legislators, and other policymakers must contend with when they are confronted with plans or proposals to adopt a transactive energy system. Most of these questions do not have simple, definitive answers, 
and the paper provides advice to regulators on how to develop answers that can guide their plans, as well as their overall decision to proceed with the proposed system or not. The questions grouped by topic are listed below.

- Managing the Transition to Transactive Energy

o What specific legislation/regulations will be required to advance along the pathway to a transactive electricity system?

o Who will bear the risk and responsibility of stranded investments? Should this be mitigated, and, if so, how?

o How should infrastructure investments to enable prosumer participation in electricity markets be evaluated?

o How can demonstration projects be most effectively used to support transition to TE?

- Market Design

o Who should be allowed to participate in a TE system? What entities will be necessary to make such a system work? Which stakeholders should be represented to make such a system fair?

o What must be done (e.g., establishing uniform contracts) to enable competitive trading of bilateral contracts?

o How should distributed energy and unbundled services be valued and priced?

o What tools and services must be available to smaller customers to enable them to participate in the TE market along with larger customers, and derive maximum benefit from the system?

o What products should be traded in TE markets (e.g., energy, capacity, kVAR, etc.)?

o When will I know that it is time to adopt locational pricing? What will be the justifications for doing so?

o How will the wholesale/retail interface be managed?

- Ongoing Market Monitoring and Evaluation

o What are the potential (negative) unintended consequences of transactive energy?

o What criteria should regulators use to evaluate the success/effectiveness of proposed or operational transactive energy systems?

- Consumer Protection

o How do I protect traditional consumers who do not want any involvement in "transactive" energy? Is there an effective way to derive the benefits of transactive energy for those consumers and prosumers who do want to engage in it, while limiting the exposure for those who don't?

o What implementation process and safeguards will be necessary to ensure that the market is operated transparently and equitably, without market power abuses?

The intent of the paper is not to be exhaustive or final, but rather to maintain an ongoing discussion of these questions and others to advance business and regulatory models for TE. The ongoing work of this team has been brought into the Smart Electric Power Alliance (SEPA) TE Working Group. 


\subsection{Reference Grid and Scenarios for TE Simulation Team}

\section{Team Members and Goals}

- Leader: Khaled Masri, National Electrical Manufacturers Association

- Participating organizations: Navigant Consulting Inc., Carnegie Mellon University, MITRE Corporation, Dartmouth University

- Goal: To develop a reference grid design and interoperability requirements that will enable testing of TE approaches using different simulation tools while producing results that can be compared for a set of agreed upon scenarios.

\section{Team Results}

The Reference Grid and Scenarios team developed a work plan that laid out the key elements needed for facilitating comparative TE simulations for the TE Challenge. These elements included:

- Identification of available reference grid data and selection of a grid useful for TE simulations;

- Selection of standard formats for representing the reference grid data and minimum data elements for definition of the reference grid;

- Consideration of TE scenarios that could be modeled on this reference grid, and selection of a baseline scenario along with event scenarios to be added; and

- Defined metrics to support inter-comparisons of results from teams simulating different TE systems on the same reference grid and scenario.

The team reviewed and considered grid models used by members in previous simulations as well as publicly available reference grid models. The team also considered a range of TE scenarios that might be used for TE Challenge team simulations. This included review and feedback on a set of TE use cases that was under development within the Smart Grid Interoperability Panel TE Coordination Group. The final results were published in a technical paper, Transactive Energy Application Landscape Scenarios [6]. The team developed a draft definition of data elements for a reference grid, and draft metrics for simulation evaluation. These results contributed to a cross-cutting effort to develop a common abstract component model to aid the development of co-simulation tools for exploring possible TE approaches (see 3.8).

\subsection{Co-Simulation Platforms Team}

\section{Team Members and Goals}

- Leaders: Himanshu Neema, Vanderbilt University, and Marija Ilic, Carnegie Mellon University

- Participating organizations: PNNL, University of Maryland, IBM Inc.

- Goals: Create a common co-simulation platform for integrating and coordinating across a diverse suite of modeling and simulation tools, and conducting integrated experiments. This goal was further specified to:

o Define TE model components list;

- Define minimum interfaces necessary for each component, where an interface is independent of any intended experiment; 
o Agree on a base experiment orchestration (e.g., UML sequence diagram showing the steps and sequence of various steps for a generic co-simulation experiment); and

o Define a reference use case scenario.

\section{Team Results}

The team members took initial steps necessary to align and guide the work outlined above. They worked to analyze the components and internal interfaces of their own co-simulation tools in relationship to other teams' tools. Vanderbilt worked with PNNL to develop an interface from the PNNL grid simulation tool (GridLAB-D) to the Vanderbilt High Level Architecture (HLA)-based co-simulation framework (called C2WT). CMU worked to analyze the possible ways that their dynamic grid simulation tool (Smart Grid in a Room Simulator) could also be connected in that framework with an HLA interface.

In late spring 2016, the idea was conceived of a focused effort involving members of both the Reference Grid and Scenarios team and the Co-simulation Platforms team that would combine the work efforts of both teams and drive toward a set of deliverables that could be used for TE Challenge comparative simulations. The results are presented in 3.8.

\subsection{Common Transactive Services}

\section{Team Members and Goals}

- Leaders: William Cox, Energy Mashup Lab and Shawn Chandler, Navigant

- Participating organizations: Alliander, Maximum Control Technologies USA, TeMix Inc., Caltech Resnick Institute, Netherlands Organisation for Applied Scientific Research (TNO)

- Goal: Seek semantic alignment between existing transactive messaging approaches in order to enable interoperable exchanges between TE systems.

\section{Team Results}

The Common Transactive Services team examined existing TE approaches in order to extract the foundational TE services that might be used for any TE system's communications. The driver for this work was the premise that, as TE system deployments proliferate, they need to work together at boundaries between systems. The approach was to use services defined in the Organization for the Advancement of Structured Information Standards (OASIS) Energy Interoperation standard as a bridge between systems at the connection points. The results are documented in a paper, "Common Transactive Services” [8] and summarized below.

\section{Common Transactive Services Paper}

As transactive energy system deployments proliferate, they need to work together at boundaries between systems. The goal of this paper was to identify a set of common transactive services that are both minimal and complete with respect to a set of deployed transactive energy systems. These services would provide common semantics to enable information mapping between the different transactive systems, for example, a microgrid operating a transactive market using one TE system participating in a larger market operating with a different TE system. The services could also help to define a common simulation interface for different transactive systems. 
The paper examined four transactive data models:

- Energy Interoperation - an OASIS standard that has been profiled to define TeMix and OpenADR;

- Pacific Northwest Demonstration Project - a Department of Energy sponsored project that used incentive signals for balancing power;

- PowerMatcher - a European open-source platform for balancing power among devices and subsystems; and

- IEC 62325 family of standards - covering IEC market implementations and used in European markets.

The Energy Interoperation, Pacific Northwest Demonstration Project and PowerMatcher data models have been implemented in TE system demonstrations. The IEC 62325 data model is a standard used primarily for European wholesale market operations, but not for TE implementation at the retail level (i.e., distribution system level).

The team proposed the following characteristics for the common transactive services:

- Clearly defined and standardized service requests and responses;

- Extensibility and adaptability, with standard models for price and product definition;

- Open (free to read and use);

- Amenable to open source implementations; and

- As simple and minimal as possible.

The selected common services were taken from the Energy Interoperation standard and are listed in Table 1. Full definitions are given in the Energy Interoperation standard [9].

Table 1 Common Transactive Services

\begin{tabular}{|l|l|l|}
\hline $\begin{array}{l}\text { Common } \\
\text { Transactive } \\
\text { Service }\end{array}$ & Description & $\begin{array}{l}\text { Names Used in Other } \\
\text { Transactive Systems }\end{array}$ \\
\hline Quote & $\begin{array}{l}\text { Provide or request a price quotation on a } \\
\text { product }\end{array}$ & Price Quote \\
\hline Tender & $\begin{array}{l}\text { Make a tender to buy or sell a product. } \\
\text { Tenders may be binding or non-binding. }\end{array}$ & Offer, Bid \\
\hline Transaction & $\begin{array}{l}\text { Accept a Tender, agreeing to and creating a } \\
\text { Transaction binding on the parties. }\end{array}$ & $\begin{array}{l}\text { Acceptance, Contract, } \\
\text { Clearing }\end{array}$ \\
\hline Delivery & Meter the actual delivery quantity & $\begin{array}{l}\text { Verify, Certify, Meter, } \\
\text { Read Meter }\end{array}$ \\
\hline
\end{tabular}

The Quote and Tender services lead to a Transaction, which is an energy-economic exchange that specifies product (e.g., energy, transport, reserves, frequency support), delivery location, and time interval for the agreed price.

The paper presents a review of the four transactive systems listed above, with references. Each transactive system is described along with details about the mapping to the proposed common transactive services. 


\subsection{TE Demo for Microgrid Energy Management Team}

\section{Team Members and Goals}

- Leader: Jennifer Worrall, Cleanspark Inc.

- Participating organizations: TeMix Inc., University of Oklahoma, The Energy Mashup Lab, General Microgrids, Tata Consultancy, Massachusetts Institute of Technology, ABB Inc., Navigant Consulting Inc., Businnovation, Dartmouth University, OATI Inc., Alstom Inc., Growing Energy Labs, Inc.

- Goals:

o Demonstrate transactive control for energy management in microgrid systems;

o Develop software and test it in real microgrid implementation; and

o Strive for scalability — account for devices inside of building up to wholesale market scale.

\section{Team Results}

The Microgrid Demonstration team focused on applying transactive mechanisms to the management of microgrids. They examined the scenario of a microgrid within a larger grid, where the microgrid energy resources are managed via transactive market methods. The approach was to adopt an agile software development process that included use case elicitation, requirement specification, design, and testing. The result was the development of a reference architecture that includes use cases, specification of software components, and a development roadmap for a microgrid-based TE system. The work of the team was documented in the paper "Transactive Energy Challenge Energy Management in Microgrid Systems" [9] and is summarized below.

\section{Transactive Energy Challenge Energy Management in Microgrid Systems Paper}

The paper introduction notes the growth of microgrid implementations worldwide, driven by several factors including: the increased availability and affordability of energy storage and renewable energy sources; advancement of interconnection standards; and proliferation of rural electrification projects (e.g., in India). At the same time, management of microgrids is becoming more complex with a shift to intermittent renewables (away from fossil-fueled generators) and growth in number of smaller flexible loads. This shift to microgrids with growing complexity is a driver for the development of transactive market methods for microgrid control. The team collaborated with the Common Transactive Services team (see 3.5) to develop a reference architecture for a microgrid TE system based on services defined in the OASIS Energy Interoperation standard [10] that would enable self-optimization of resources.

The team identified three actors in the TE system: Market Participant, Market Maker, and Market Facilitator. These actors communicate and perform their respective functions by applying sequences of defined actions described in Table 2. 
Table 2. Actions of the TE System Actors.

\begin{tabular}{|l|l|}
\hline Action & Description \\
\hline Enroll & $\begin{array}{l}\text { Market Participant registers with the Market Facilitator in order to } \\
\text { begin receiving and sending tenders. }\end{array}$ \\
\hline Tender tender & $\begin{array}{l}\text { Submit a new tender or accept/reject an existing tender as the } \\
\text { counter party. }\end{array}$ \\
\hline Determine price & $\begin{array}{l}\text { If submitting a new tender, determine the ask price; otherwise, } \\
\text { determine if ask price is acceptable. }\end{array}$ \\
\hline Accept transaction & $\begin{array}{l}\text { If price is acceptable, accept tender as a transaction and schedule } \\
\text { Market Participant accordingly. }\end{array}$ \\
\hline Provide power & $\begin{array}{l}\text { At the start time recorded in the transaction, the Market Participant } \\
\text { modifies operations to accept the requested power from the local } \\
\text { microgrid. }\end{array}$ \\
\hline $\begin{array}{l}\text { At the start time in the transaction, the Market Participant modifies } \\
\text { operations to provide the requested power to the local microgrid. } \\
\text { This power may be provided by load reduction, overlaying a } \\
\text { previous transaction to accept power. }\end{array}$ \\
\hline Deliver report & $\begin{array}{l}\text { At the completion of the transaction or on another agreed-upon } \\
\text { interval, the Market Participant reports telemetry data documenting } \\
\text { the power generated or accepted. }\end{array}$ \\
\hline
\end{tabular}

The paper then specifies two software packages, each with a collection of components required for implementation. The Energy Services Interface package is composed of the base communications framework in addition to the Market Participant and Market Maker actor functionality. The Market Participant has components for determining price acceptability, forecasting generation or demand, scheduling, control, and reporting of actual power use or generation. The Market Maker helps to increase market liquidity by frequently issuing a series of small buy/sell forward tenders. The Market Facilitator package provides enrollment (to authenticate and authorize Market Participants), validates tenders and transactions, records transactions in a ledger, and settles transactions.

While time constraints did not enable the team to build an implementation, this report provides a foundation for any future effort to architect a transactive solution for microgrid energy management.

\subsection{Transactive Automated Demand Response team}

\section{Team Members and Goals}

- Leader: Rolf Bienert, OpenADR Alliance

- Participating organizations: CleanSpark, The Energy Mashup Lab, Alliander

- Goal: Add TE services to the existing OpenADR demand response protocol, in collaboration with the established OpenADR Alliance.

\section{Team Results}

The Transactive Automated Demand Response (ADR) team initiated an effort to add transactive services to the OpenADR protocol [11] with the input of TE Challenge 
participants. OpenADR is an established demand response protocol used worldwide, and the OpenADR Alliance maintains the implementation profiles and provides testing and certification [11]. OpenADR includes a set of services that are profiled from OASIS Energy Interoperation. In order to accomplish the goal of adding TE services to the established OpenADR protocol, the team decided to set up a working group within the OpenADR Alliance. They agreed to take the work of the Common Transactive Services team with the defined four transactive services and provide this as input to the working group. However, as of the publication of this report, the work is still ongoing within the OpenADR Alliance.

\subsection{PowerMatcher IoT Team}

\section{Team Members and Goals}

- Leader: William Miller, Maximum Control Technologies (MaCT)-USA

- Participating organizations: Alliander, TNO, State University of New York (SUNY)/Buffalo State, Rowan State, Carnegie Mellon University

- Goal: Demonstrate the benefits to the U.S. electric grid of a market approach using PowerMatcher.

\section{Team Results}

PowerMatcher [12] is a transactive protocol developed originally at the TNO Netherlands for enabling power management among home devices and groups of homes. It has been used in utility demonstrations in several European cities and standardization activities are now proceeding in Europe. The PowerMatcher technology is a system for devices (or their agents) to bid a demand curve into an auction on a 5 min market cycle. The auctioneer sets the market price based on bids and communicates back to device agents. Devices then respond to balance supply and demand. Concentrator nodes allow for aggregation and thus scalability.

The PowerMatcher IoT team made steady progress recruiting team members for a virtual PowerMatcher testbed implementation in the northeast U.S. and Canada. The testbed creation was not complete when the TE Challenge Phase I finished. However, the PowerMatcher IoT testbed work was migrated to the NIST Global City Teams Challenge [13], and the team project continues as of time of this report. The team plan has shifted focus to now being centered in Manila, Philippines with a combination of PowerMatcher networks to manage demand on the electric system together with block chain technology to track renewable energy production.

\subsection{Development of a Common TE Abstract Component Model}

As the work of the Challenge progressed, it became apparent that, by leveraging the results from the Reference Grid and Scenarios and the Co-Simulation Platforms teams, it might be possible to set the stage for a Phase II of the Challenge where simulations of a particular scenario on a specific grid might be conducted in a way that the results can be directly compared. A cross-cutting team with representatives from Carnegie Mellon, NIST, PNNL, and Vanderbilt was formed to work out the details. NIST served as both convener and model manager. Other representatives provided TE co-simulation expertise. The team worked through the summer of 2016 to select a specific scenario and a reference grid. It also developed an abstract model of the simulation components needed to carry out a TE simulation. 
The idea behind the model was that a common abstract representation of the functionality and interfaces between key simulation components could facilitate co-simulation opportunities and define common output metrics that enable meaningful comparisons. Standard interfaces would enable existing tools with different component functionality to be linked in co-simulation. The resulting TE Abstract Component Model [7] was defined using the Unified Modeling Language (UML).

The model is composed of six components which can be duplicated and combined in flexible ways to represent the details of a comprehensive range of TE scenarios.

- Resource - represents loads, storage, and generation involved in transactive energy.

- Local Controller - is intimately bound to a single resource and can be instructed by a Supervisory Controller.

- Supervisory Controller - has a one-to-many relationship with Local Controllers and Resources to administer a facility-wide or enterprise-wide strategy for reacting to TE.

- Transactive Agent - is a participant in an economic bidding system - includes both customer representatives and central market components.

- Weather - provides the environmental data that drive the dynamics of the TE scenario.

- Grid - comprises the distribution system model, including its source of bulk power and internal distributed controls.

Figure 1 illustrates how these components can be assembled in various ways to represent the diversity of actors that might be found in transactive energy systems. Grid link 1 has a transactive appliance that can bid directly into a market. It is represented by the combination of the abstract components Load, Local Controller, Supervisory Controller and Transactive Agent. On grid link 2, a distribution system operator provides a market interface for a bulk generator. The distribution system operator is represented by a combination of the Grid and Transactive Agent components. The bulk generator is represented by the Resource and Local Controller components. Grid link 3 represents a facility management system with a single supervisory controller transacting energy on behalf of an industrial customer with distributed energy resources and loads to administer. In this case the abstract components used are Resource, Local Controller, Supervisory Controller, and Transactive Agent. 


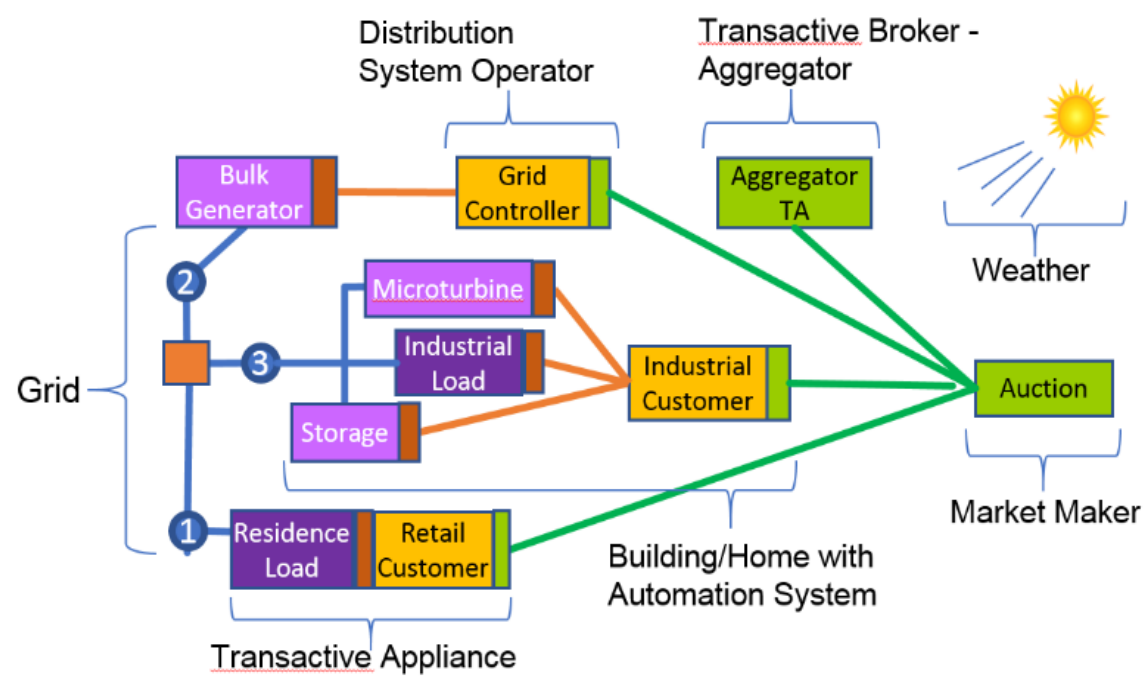

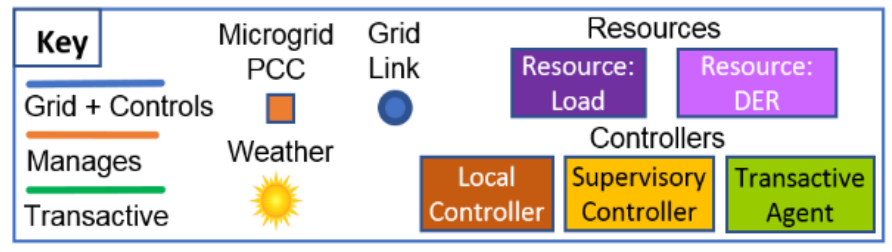

Figure 1. Notional topology of a TE simulation [7]

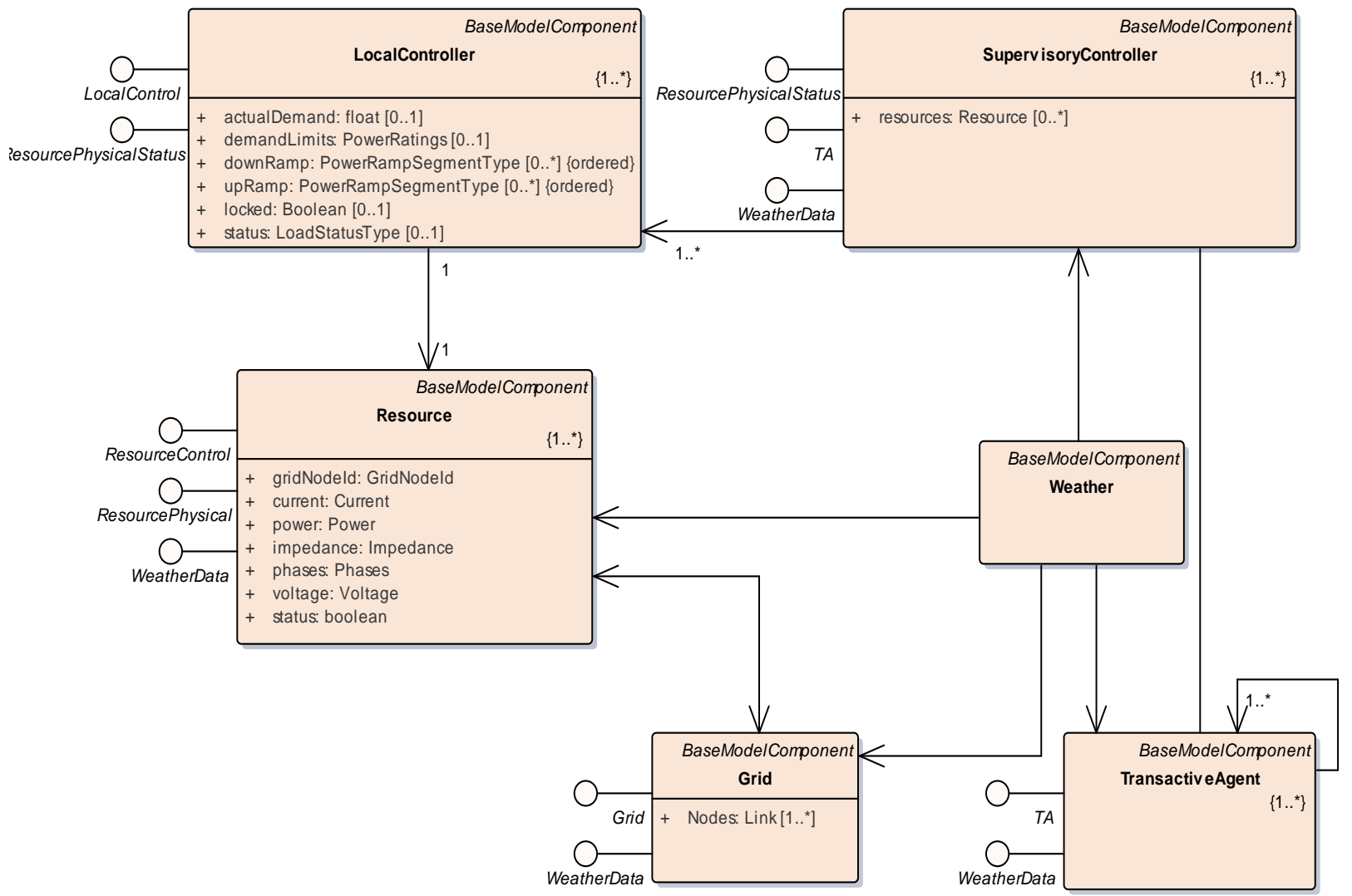

Figure 2. Core modeling components of Abstract Component Model [7] 
Figure 2 is a UML diagram derived from the model showing the core components and exposing the logical interfaces needed for transactive energy simulations. The interfaces are represented by the circle nodes extending to the left of each rectangular component body. Some of the attributes of the components are shown within the component bodies. Full details of the model along with examples and other supporting information about how to apply it can be found in [7].

\section{Summary}

The TE Challenge Phase I succeeded in bringing together industry experts concerned about improving the ability to effectively model potential TE systems. The results include the development of a set of example TE use cases, development of an Abstract Component Model that can facilitate combining tools in a co-simulation environment, an analysis of common transactive services, development of a reference architecture for microgrid-based $\mathrm{TE}$, and analysis of the current TE business and regulatory environment. Phase I created a foundation for a Phase II Challenge focused on using multiple tools or simulation platforms to simulate different TE approaches applied to a particular use case on a common grid with results that can be directly compared.

\section{References}

[1] GridWise Architecture Council (2015) GridWise Transactive Energy Framework 1.0. http://www.gridwiseac.org/pdfs/te_framework_report_pnnl-22946.pdf.

[2] Energetics Inc. (2015) Transactive Energy Challenge Kickoff Workshop Summary Report. https://www.nist.gov/document-18647.

[3] NIST (2015) Simulation tools and co-simulation platforms, TE Challenge collaboration site Tool Chest. https://pages.nist.gov/TEChallenge/toolchest/.

[4] Cazalet, E., De Martini, P., Price, J., Woychik, E., Caldwell, J. (2016) Transactive Energy Models. TE Challenge Business and Regulatory Models team paper. https://s3.amazonaws.com/nist-sgcps/TEChallenge/library/TE-Bus-Reg-Models-Paper20160920.pdf.

[5] Caldwell, J., Cazalet, E., Cox., W., De Martini, P., Dobriansky, L., Heitmann, P., Price, J., Radloff, G., Rahimi, F., Woychik, E. (2017) The Policymaker's Toolkit--Vital Questions to be Addressed about Proposed Transactive Energy Systems. https://s3.amazonaws.com/nist-sgcps/TEChallenge/Library/BusRegTeamPolicymakerToolkitPaper-Jun2017.pdf.

[6] Holmberg, D., Hardin, D., Cunningham, R., Melton, R., Widergren, S. (2016) Transactive Energy Application Landscape Scenarios. SGIP Technical Paper. http://www.sgip.org/wp-content/uploads/SGIP_White_Paper_TE_Application_ Landscape_Scenarios_12-15-2016_FINAL.pdf.

[7] Burns, M., Song, E., Holmberg, D (2018), The Transactive Energy Challenge Abstract Component Model, NIST Special Pub. 1900-602, https://doi.org/10.6028/NIST.SP.1900602.

[8] Cox, W., et. al. (2016) Common Transactive Services. https://s3.amazonaws.com/nistsgcps/TEChallenge/Library/CTS-Report-20160502.pdf. 
[9] Worrall, J., Cazalet, E., Cox, W., Rajagopal, N., Nudell, T., Heitmann, P. (2016) Transactive Energy Challenge Energy Management in Microgrid Systems. Proceedings of the TE Systems Conference and Workshop, Portland, OR, May 2016. http://www.gridwiseac.org/pdfs/tes_2016/tes2016.pdf.

[10] OASIS Standard (2011-2014) Energy Interoperation 1.0. http://docs.oasisopen.org/energyinterop/ei/v1.0/energyinterop-v1.0.html.

[11] OpenADR Alliance, http://www.openadr.org.

[12] PowerMatcher Technology, http://flexiblepower.github.io/technology/powermatcher/.

[13] Global Cities Team Challenge (2017) PowerMatcher Blockchain Action Cluster in the Philippines. Presented at the August 2017 GCTC Expo.

https://pages.nist.gov/GCTC/action-clusters/. 